\title{
The Cartesian Product of Hypergraphs
}

Lydia Gringmann AND INTERDISCIPLINARY GROUP, DEPARTMENT OF COMPUTER SCIENCE AND INTERDISCIPLINARY CENTER FOR BIOINFORMATICS UNIVERSITY OF LEIPZIG, HÄRTELSTRASSE 16-18, D-04107 LEIPZIG, GERMANY E-MAIL: GLYDIA@BIOINF.UNI-LEIPZIG.DE

Marc Hellmuth

MAX PLANCK INSTITUTE FOR MATHEMATICS IN THE SCIENCES INSELSTRASSE 22, D-04103 LEIPZIG, GERMANY

BIOINFORMATICS GROUP, DEPARTMENT OF COMPUTER SCIENCE AND INTERDISCIPLINARY CENTER FOR BIOINFORMATICS UNIVERSITY OF LEIPZIG, HÄRTELSTRASSE 16-18, D-04107 LEIPZIG, GERMANY E-MAIL:MARC@BIOINF.UNI-LEIPZIG.DE

Peter F. Stadler

BIOINFORMATICS GROUP, DEPARTMENT OF COMPUTER SCIENCE AND INTERDISCIPLINARY CENTER FOR BIOINFORMATICS UNIVERSITY OF LEIPZIG, HÄRTELSTRASSE 16-18, D-04107 LEIPZIG, GERMANY

MAX PLANCK INSTITUTE FOR MATHEMATICS IN THE SCIENCES INSELSTRASSE 22, D-04103 LEIPZIG, GERMANY

RNOMICS GROUP, FRAUNHOFER INSTITUT FÜR ZELLTHERAPIE UND IMMUNOLOGIE, DEUTSCHER PLATZ 5E, D-04103 LEIPZIG, GERMANY

DEPARTMENT OF THEORETICAL CHEMISTRY, UNIVERSITY OF VIENNA, WÄHRINGERSTRAßE 17, A-1090 WIEN, AUSTRIA

SANTA FE INSTITUTE, 1399 HYDE PARK RD., SANTA FE, NM87501, USA E-MAIL: STUDLA@BIOINF.UNI-LEIPZIG.DE 


\section{ABSTRACT}

We show that every simple, (weakly) connected, possibly directed and infinite, hypergraph has a unique prime factor decomposition with respect to the (weak) Cartesian product, even if it has infinitely many factors. This generalizes previous results for graphs and undirected hypergraphs to directed and infinite hypergraphs. The proof adopts the strategy outlined by Imrich and Žerovnik for the case of graphs and introduced the notion of diagonal-free grids as a replacement of the chord-free 4-cycles that play a crucial role in the case of graphs. This leads to a generalization of relation $\delta$ on the arc set, whose convex hull is shown to coincide with the product relation of the prime factorization.

Keywords: directed Hypergraph, Hypergraph, weak Cartesian Product, Prime Factor Decomposition, grid property

\section{INTRODUCTION}

Directed hypergraphs are the common generalization of both directed graphs and (undirected) hypergraphs. A (directed) hypergraph $H=(V, \mathcal{E})$ consists of a vertex set $V$ and a family of (directed) hyperedges or hyperarcs $\mathcal{E}$. Each hyperedge $E \in \mathcal{E}$ is an ordered pair of sets of vertices $E=(T(E), H(E)) \neq(\emptyset, \emptyset)$. The sets $T(E) \subseteq V$ and $H(E) \subseteq V$ are called the tail and head of $E$, respectively. To avoid the risk of confusion we will sometimes write $V(H)$ and $\mathcal{E}(H)$ for the vertex set and the arc set of a hypergraph $H$.

A hypergraph $H=(V, \mathcal{E})$ is undirected if $T(E)=H(E)$ for all $E \in \mathcal{E}$. Most of the literature on hypergraphs is concerned with undirected hypergraphs [1], surveys on directed hypergraphs can be found in $[7,8]$. Directed hypergraphs have been used as model for complex networks in biology and chemistry [15]. For instance a chemical reaction is naturally represented as a hyperedge $E$ where $T(E)$ lists the educts and $H(E)$ the products of the chemical transformation.

We say a hypergraph is finite if its vertex set and its edge set is finite. A hypergraph that is not finite is said to be infinite. A hypergraph $H=(V, \mathcal{E})$ is called simple, if (i) $|T(E) \cup H(E)|>1$ for all $E \in \mathcal{E}$ and (ii) there are no two distinct $\operatorname{arcs} E, E^{\prime} \in \mathcal{E}$ such that $T(E)=T\left(E^{\prime}\right)$ and $H(E)=H\left(E^{\prime}\right)$. A hypergraph is a directed graph if $|T(E)|=|H(E)|=1$ for all $E \in \mathcal{E}$.

We will be concerned with products of hypergraphs, more precisely with the most fundamental question that arises in this context: Does a hypergraph have a unique decomposition into prime factors? The answer to this question depends on the definition of the product. In the case of graphs, the product structures are quite well understood $[11]$.

Several notions of hypergraph products have been studied in the literature, usually for restricted versions of undirected hypergraphs, see e.g. [2, 6, 9, 20]. The most commonly studied hypergraph product is the direct product, which consists of the Cartesian product of vertex sets, and the Cartesian (set) products of the hyperedges [3, 5, 18, 17]. For direct 
CARTESIAN PRODUCT OF HYPERGRAPHS 3

products of $N$-systems, i.e., directed hypergraphs satisfying $|T(E)|=1$ and $T(E) \subseteq$ $H(E)$ for all $E \in \mathcal{E}$, a prime factor theorem was proved in [13].

In this contribution we will focus on the Cartesian product of finite and infinite directed hypergraphs with finitely or infinitely many factors. The Cartesian graph product was introduced by Gert Sabidussi [19], who showed that connected graphs have a unique Cartesian prime factor decomposition. This result was generalized by Wilfried Imrich [9] to simple undirected hypergraphs:

Definition. Let $\left\{H_{i} \mid i \in I\right\}$ be a family of (finite or infinite), but undirected hypergraphs. The Cartesian product $H=\square_{i \in I} H_{i}$ is defined as follows:

$$
\begin{aligned}
& V(H)=\underset{i \in I}{\times} V\left(H_{i}\right) \\
& \mathcal{E}(H)=\left\{E \subseteq V(H) \mid p_{j}(E) \in \mathcal{E}\left(H_{j}\right) \text { for exactly one } j \in I, \text { and }\left|p_{i}(E)\right|=1 \text { for } i \neq j\right\},
\end{aligned}
$$

where, for $j \in I, p_{j}: V(H) \rightarrow V\left(H_{j}\right)$ is the projection of the Cartesian product of the vertex sets into $V\left(H_{j}\right)$. The value $p_{j}(v)$ is also called $j$-th coordinate of vertex $v$.

The Cartesian product of undirected hypergraphs is also considered for example in $[3,4,5]$ A factorization algorithm for so-called conformal hypergraphs, a rather small class of finite and connected hypergraphs, with respect to the Cartesian product, is described in [4].

While the Cartesian product hypergraph of finitely many connected hypergraphs is connected, whether they are finite or not, this does not hold for the product of infinitely many hypergraphs: In this case, there are vertices that differ in infinitely many coordinates and hence are not connected by a path of finite length. As in the case of graphs [19], an infinite connected hypergraph that has infinitely many Cartesian prime factors cannot be the Cartesian product of its factors, but a connected component of this Cartesian product [10]. This gives rise to the notion of a weak Cartesian product:

Definition. [10] Let $\left\{H_{i} \mid i \in I\right\}$ be a family of hypergraphs and let $a_{i} \in V\left(H_{i}\right)$ for $i \in I$. The weak Cartesian product $H=\square_{i \in I}\left(H_{i}, a_{i}\right)$ of the "rooted" hypergraphs $\left(H_{i}, a_{i}\right)$ is defined by

$$
\begin{aligned}
& V(H)=\left\{v \in \underset{i \in I}{\times} V\left(H_{i}\right) \mid p_{i}(v) \neq a_{i} \text { for at most finitely many } i \in I\right\} \\
& \mathcal{E}(H)=\left\{E \subseteq V(H) \mid p_{j}(E) \in \mathcal{E}\left(H_{j}\right) \text { for exactly one } j \in I, \text { and }\left|p_{i}(E)\right|=1 \text { for } i \neq j\right\} .
\end{aligned}
$$

For finite index sets $I$, the weak Cartesian product does not depend on the choice of the $a_{i}$ and coincides with the usual Cartesian product. If $I$ is infinite, it is the connected component of the Cartesian product containing $a=\left(a_{i}\right)_{i \in I}$. Every connected graph and undirected hypergraph has a unique representation as a weak Cartesian product $[16,10]$.

Here we extend and generalize these results further and show that every connected directed hypergraph has a unique prime factor decomposition with respect to the (weak) 


\section{JOURNAL OF GRAPH THEORY}

Cartesian product. Instead of following the proof strategies of the classical papers, we adopt the approach of Imrich and Žerovnik [14] that constructs a product relation $\sigma$ starting from simpler relations on the edge set $E$. In the case of graphs, the Square Property [12] plays a central role as technical device. The Grid Property, which is introduced here, serves as generalization of this construction. Together with a generalization of the relation $\delta$, we arrive at our main results:

Theorem 1. Let $\gamma$ be a convex equivalence relation on the $\operatorname{arc}$ set $\mathcal{E}(H)$ of a connected simple hypergraph $H$ which satisfies the grid property. Then $\gamma$ induces a factorization of $H$ with respect to the weak Cartesian product.

Theorem 2. Every connected simple hypergraph has a unique representation as a weak Cartesian product.

Theorem 3. The product relation $\sigma$ corresponding to the unique prime factor decomposition with respect to the weak Cartesian product of a connected simple hypergraph $H$ equals the convex hull $\mathcal{C}(\delta)$ of the relation $\delta$.

Since the Cartesian and the weak Cartesian product coincides for a finite number of factors, we also obtain the following corollaries.

Corollary. The prime factor decomposition of a connected hypergraph with finitely many factors with respect to the Cartesian product is unique in the class of simple hypergraphs.

Corollary. The prime factor decomposition of a connected hypergraph with respect to the Cartesian product is unique in the class of finite simple hypergraphs.

\section{PRELIMINARIES}

As far as possible, we follow the notation and terminology of Berge's classical book on hypergraphs [1], although our hypergraphs will in general be directed, $T(E) \neq H(E)$ for some $E \in \mathcal{E}$. For simplicity, we will refer to hyperarcs as arcs.

Two $\operatorname{arcs} E, E^{\prime} \in \mathcal{E}(H)$ of a hypergraph $H$ are incident if $E \cap E^{\prime} \neq \emptyset$, i.e., if there is a vertex that is contained in both arcs, independent of the directions. Two vertices $x, y \in V$ are adjacent if there is an arc $E$ containing them, i.e., $x, y \in T(E) \cup H(E)$, again without regard of direction.

Concepts of sub-hypergraphs, paths, etc., are defined in the appendix to make this contribution self-consistent.

For the set product $V=\times_{i \in I} V_{i}$ we define the projection $p_{j}: V \rightarrow V_{j}$ by $\left(v_{1}, v_{2}, \ldots, v_{j}, \ldots\right) \mapsto v_{j}$. For subsets of $V$ and ordered tuples of elements of $V$, the projection is defined element-wise. For example, for a hypergraph $H=\left(\times_{i \in I} V_{i}, \mathcal{E}\right)$ we 
have

$$
p_{j}(E)=p_{j}(T(E), H(E))=\left(p_{j}(T(E)), p_{j}(H(E))\right)=\left(\bigcup_{v \in T(E)} p_{j}(v), \bigcup_{w \in H(E)} p_{j}(w)\right)
$$

By abuse of notation, we will write

$$
\left|p_{j}(E)\right|:=\left|p_{j}(T(E)) \cup p_{j}(T(H))\right|
$$

i.e., $\left|p_{j}(E)\right|$ refers to the cardinality of the union of the projections of head and tail of the arc $E$.

Definition. Let $H_{i}, i \in I$ be hypergraphs. The Cartesian product $\square_{i \in I} H_{i}$ has the following vertex and arc sets:

(1) $V\left(\square_{i \in I} H_{i}\right)=\times_{i \in I} V\left(H_{i}\right)$,

(2) and $E=(T(E), H(E))$ is an arc in $\square_{i \in I} H_{i}$ if and only if there is a $j \in I$, such that

(i) $p_{j}(E)=p_{j}((T(E), H(E)))=\left(T\left(p_{j}(E)\right), H\left(p_{j}(E)\right)\right) \in \mathcal{E}\left(H_{j}\right)$ and

(ii) $\left|p_{i}(E)\right|=1$ for all $i \neq j$.

Figure 1 shows an example of a Cartesian product of two hypergraphs.
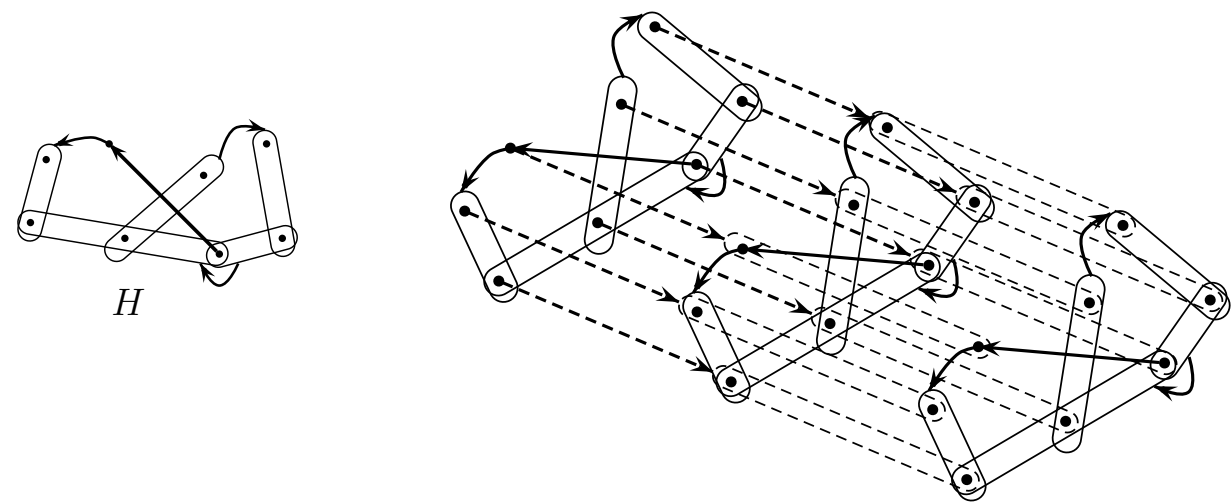

FIGURE 1. Hypergraph $H$ and Cartesian product $H \square E_{1,2}$, where $E_{1,2}$ is the hypergraph consisting of a single arc of three vertices such that $\left|T\left(E_{1,2}\right)\right|=1$ and $\left|H\left(E_{1,2}\right)\right|=2$.

Lemma 1. The Cartesian Product $H=\square_{i \in I} H_{i}$ of hypergraphs $H_{i}$ is an undirected hypergraph if and only if all of its factors are undirected.

Proof. The assertion follows directly from equ.(1).

By construction, the Cartesian product is associative, commutative, distributive w.r.t. the disjoint union and has the trivial one vertex hypergraph $K_{1}$ without arcs as unit. 
Lemma 2. The Cartesian product $H=\square_{i=1}^{n} H_{i}$ of finitely many hypergraphs $H_{i}$ is connected if and only if all of its factors $H_{i}$ are connected.

Proof. Because of associativity and commutativity it suffices to show the assertion for two factors, hence let $H=H_{1} \square H_{2}$.

First, assume that $H_{1}$ and $H_{2}$ are connected. Let $v=(x, y)$ and $v^{\prime}=\left(x^{\prime}, y^{\prime}\right)$ be two arbitrary vertices in $V(H)$. Consider a path $P_{x x^{\prime}}=\left(E_{1}, \ldots, E_{r}\right)$ from $x$ to $x^{\prime}$ in $H_{1}$ and a path $P_{y y^{\prime}}=\left(F_{1}, \ldots, F_{s}\right)$ from $y$ to $y^{\prime}$ in $H_{2}$. Then $\left(E_{1} \times\{y\}, \ldots, E_{r} \times\{y\}\right)$ is a path from $(x, y)$ to $\left(x^{\prime}, y\right)$ in $H$ and $\left(\left\{x^{\prime}\right\} \times F_{1}, \ldots,\left\{x^{\prime}\right\} \times F_{s}\right)$ is a path from $\left(x^{\prime}, y\right)$ to $\left(x^{\prime}, y^{\prime}\right)$ in $H$. Hence, $\left(E_{1} \times\{y\}, \ldots, E_{r} \times\{y\},\left\{x^{\prime}\right\} \times F_{1}, \ldots,\left\{x^{\prime}\right\} \times F_{s}\right)$ is a path from $v$ to $v^{\prime}$ in $H$.

W.l.o.g., suppose that $H_{1}$ is not connected, i.e., it can be written in the form $H_{1}=$ $H_{1}^{\prime}+H_{1}^{\prime \prime}$. Since the Cartesian product is distributive w.r.t. the disjoint union, we have $H=H_{1}^{\prime} \square H_{2}+H_{1}^{\prime \prime} \square H_{2}$. Hence $H$ is the disjoint union of two hypergraphs, i.e., $H$ is not connected.

Let us now turn to the weak Cartesian product. As in the undirected case, the product of finitely many connected hypergraphs is connected, whether they are finite or not, but the product of infinitely many factors is not connected.

Definition. Let $\left\{H_{i} \mid i \in I\right\}$ be a family of hypergraphs and let $a_{i} \in V\left(H_{i}\right)$ for $i \in I$. The weak Cartesian product $H=\square_{i \in I}\left(H_{i}, a_{i}\right)$ of the hypergraphs $\left(H_{i}, a_{i}\right)$ rooted at $a_{i}$ is given by

$$
\begin{aligned}
V(H) & =\left\{v \in \underset{i \in I}{\times} V\left(H_{i}\right) \mid p_{i}(v) \neq a_{i} \text { for at most finitely many } i \in I\right\} \\
\mathcal{E}(H) & =\left\{E \subseteq V(H) \mid p_{j}(E)=p_{j}((T(E), H(E)))=\left(T\left(p_{j}(E)\right), H\left(p_{j}(E)\right)\right) \in \mathcal{E}\left(H_{j}\right)\right.
\end{aligned}
$$

for exactly one $j \in I$, and $\left|p_{i}(E)\right|=1$ for $\left.i \neq j\right\}$.

We will, in the following write $\square_{i \in I}^{a} H_{i}$ for $\square_{i \in I}\left(H_{i}, a_{i}\right)$, where $a \in V(H)$ such that $p_{i}(a)=a_{i}$ for all $i \in I$.

Again, the weak Cartesian product does not depend on the $\left(a_{i}\right)_{i \in I}$ if $I$ is finite. Moreover, it coincides with the Cartesian product if $I$ is finite.

The partial hypergraph of $H$ induced by all vertices of $H$ which differ from $w \in V(H)$ exactly in the $j$-th coordinate is isomorphic to $H_{j}$. More formally

$$
\left\langle\left\{v \in V(H) \mid p_{k}(v)=w_{k} \text { for } k \neq j\right\}\right\rangle \simeq H_{j} .
$$

We will call this partial hypergraph the $H_{j}$-layer through $w$ and denote it by $H_{j}^{w}$. The isomorphism $H_{j}^{w} \rightarrow H_{j}$ is then the projection $p_{j}$. For $u \in V\left(H_{j}^{w}\right)$ we have $H_{j}^{u}=H_{j}^{w}$ and, moreover, $V\left(H_{j}^{u}\right) \cap V\left(H_{j}^{w}\right)=\emptyset$ if and only if $u \notin V\left(H_{j}^{w}\right)$.

Lemma 2 implies the following 
Corollary. A weak Cartesian product $H=\square_{i \in I}^{a} H_{i}$ is connected if and only if all of its factors $H_{i}$ are connected. In this case, $H=\square_{i \in I}^{a} H_{i}$ is the connected component of the Cartesian product $\square_{i \in I} H_{i}$ that contains the vertex $a$.

Lemma 3. The Cartesian product $H=\square_{i \in I} H_{i}$ of hypergraphs $H_{i}$ is simple if and only if all of its factors $H_{i}$ are simple.

Proof. Let $E_{x j}$ denote an arc containing $x \in V$ and whose projection on the $j$-th factor is an arc in the $j$-th factor.

First let all $H_{i}, i \in I$ be simple and suppose $H$ is not simple. We have to examine two cases: First, suppose $H$ contains at least one loop on vertex $x$ with coordinates $x_{i}, i \in I$. Hence there is a loop in some factor $H_{j}$, contradicting that $H_{j}$ is simple. Thus, it must hold $|E| \geq 2$ for all $E \in \mathcal{E}(H)$. Second, let $E_{x j}$ and $E_{y k}^{\prime}$ be two different arcs, such that $T\left(E_{x j}\right)=T\left(E_{y k}^{\prime}\right)$ and $H\left(E_{x j}\right)=H\left(E_{y k}^{\prime}\right)$. Hence, $x \in E_{y k}^{\prime}$ and $j=k$. Thus, $p_{j}\left(T\left(E_{x j}\right)\right)=p_{k}\left(T\left(E_{y k}^{\prime}\right)\right)$, as well as $p_{j}\left(H\left(E_{x j}\right)\right)=p_{j}\left(H\left(E_{y j}^{\prime}\right)\right)$. Therefore $H_{j}$ is not simple, a contradiction.

Now assume that (at least) one of the factors is not simple; w.l.o.g. say $H_{1}$. There are two possibilities: First, assume there is an $\operatorname{arc} E \in \mathcal{E}\left(H_{1}\right)$ with $|E|=1$, say $E=\left\{x_{1}\right\}$. Hence, $\left|E_{1 x}\right|=|\{x\}|=1$ for any $x \in V(H)$ with $p_{1}(x)=x_{1}$ and $H$ would not be simple. Second, assume there are two different $\operatorname{arcs} E_{i}, E_{j} \in \mathcal{E}\left(H_{1}\right)$, such that $T\left(E_{i}\right)=T\left(E_{j}\right)$ and $H\left(E_{i}\right)=H\left(E_{j}\right)$. Then, the definition of the Cartesian product implies that there are two $\operatorname{arcs} E=E_{i} \times\{x\}$ and $E^{\prime}=E_{j} \times\{x\}$ in $H$ with $x \in \times_{l \in I \backslash\{1\}} V\left(H_{l}\right)$ such that $T(E)=T\left(E^{\prime}\right)$ and $H(E)=H\left(E^{\prime}\right)$. Hence, $\mathrm{H}$ is not simple.

Note that the weak Cartesian product $\square_{i \in I}\left(H_{i}, a_{i}\right)$ is a partial hypergraph of the Cartesian product $\square_{i \in I} H_{i}$, that is induced by $V\left(\square_{i \in I}\left(H_{i}, a_{i}\right)\right)$. Hence we have

Corollary. The weak Cartesian product $H=\square_{i \in I}\left(H_{i}, a_{i}\right)$ of hypergraphs $H_{i}$ is simple if and only if all of its factors $H_{i}$ are simple.

From here on we assume that all hypergraphs are simple.

\section{UNIQUE PRIME FACTOR DECOMPOSITION}

A hypergraph $H$ is prime with respect to the (weak) Cartesian product if it cannot be represented as the (weak) Cartesian product of two nontrivial hypergraphs. A prime factor decomposition (PFD) of $H$ is a representation as a Cartesian product $H=\square_{i \in I} H_{i}$, or as a a weak Cartesian product $H=\square_{i \in I}^{a} H_{i}$, resp., such that all factors $H_{i}, i \in I$, are prime and $H_{i} \not \varkappa K_{1}$.

In order to show that every hypergraph has a unique representation as a weak Cartesian product, we follow the strategy of Imrich and Žerovnik [14] and characterize the socalled product relations defined on the $\operatorname{arc} \operatorname{set} \mathcal{E}$ of $H$. The advantage of this approach is 
that it does not require finiteness and hence also pertains to the weak Cartesian product of infinitely many factors.

Definition. A product relation is an equivalence relation on the $\operatorname{arc}$ set $\mathcal{E}(H)$ of a (weak) Cartesian product $H=\square_{i \in I}^{a} H_{i}$ of (not necessarily prime) hypergraphs $H_{i}$ such that, for $E, F \in \mathcal{E}(H), E$ and $F$ are in relation $\gamma, E \gamma F$, if and only if there exists a $j \in I$, such that

$$
\left|p_{j}(E)\right|>1 \quad \text { and } \quad\left|p_{j}(F)\right|>1,
$$

and $\left|p_{i}(E)\right|=\left|p_{i}(F)\right|=1$ holds for all $i \neq j$.

If all factors $H_{i}$ are prime, we denote this relation by $\sigma$. Note that $E$ and $F$ are in relation $\sigma$ if and only if their vertices differ in the same coordinate w.r.t. to the PFD. Let $\Sigma_{i}, i \in I$ be the equivalence classes of $\sigma$. By construction, every connected component of a partial hypergraph generated by the arcs of an equivalence class $\Sigma_{i}$ is isomorphic to $H_{i}$. Furthermore, every union of $\sigma$-equivalence classes $\bigcup_{j \in J} \Sigma_{j}, J \subseteq I$ generates a partial hypergraph of $H$, whose connected components are isomorphic to $H_{J}:=\square_{j \in J}^{a} H_{j}$.

\section{THE GRID PROPERTY}

Definition. Let $\mathcal{G}$ be a collection of arcs of $H$ of the form $E_{a}=\left(T\left(E_{a}\right), H\left(E_{a}\right)\right)$, $a \in A$ and $F_{b}=\left(T\left(F_{b}\right), H\left(F_{b}\right)\right), b \in B$. We say that $\mathcal{G}$ is an $|A| \times|B|$-grid if, for all $a, a^{\prime} \in A$ and $b, b^{\prime} \in B$ with $a \neq a^{\prime}$ and $b \neq b^{\prime}$ the following two conditions are satisfied:

(i) $E_{a}$ and $F_{b}$ have exactly one vertex in common, i.e., $\left(T\left(E_{a}\right) \cup H\left(E_{a}\right)\right) \cap\left(T\left(F_{b}\right) \cup\right.$ $\left.H\left(F_{b}\right)\right)=\left\{z_{a b}\right\}$, and

(ia) $z_{a b} \in T\left(F_{b}\right)$ (resp. $\left.H\left(F_{b}\right)\right)$ if and only if $z_{a b^{\prime}} \in T\left(F_{b^{\prime}}\right)$ (resp. $H\left(F_{b^{\prime}}\right)$ ) for all $b^{\prime} \in B$, and

(ib) $z_{a b} \in T\left(E_{a}\right)$ (resp. $H\left(E_{a}\right)$ ) if and only if $z_{a^{\prime} b} \in T\left(E_{a^{\prime}}\right)$ (resp. $H\left(E_{a^{\prime}}\right)$ ) for all $a^{\prime} \in A$, and

(ii) $E_{a}$ and $E_{a^{\prime}}$ have no common vertex for $a \neq a^{\prime}$, i.e., $\left(T\left(E_{a}\right) \cup H\left(E_{a}\right)\right) \cap\left(T\left(E_{a}^{\prime}\right) \cup\right.$ $\left.H\left(E_{a}^{\prime}\right)\right)=\emptyset$. Analogously, $F_{b}$ and $F_{b^{\prime}}$ are disjoint for $b \neq b^{\prime}$.

An $\operatorname{arc} D \in \mathcal{E}(H)$ satisfying $z_{a b} \in D$ and $z_{a^{\prime} b^{\prime}} \in D$ for all for $a, a^{\prime} \in A$ and $b, b^{\prime} \in B$ with $a \neq a^{\prime}$ and $b \neq b^{\prime}$, is a diagonal of the $|A| \times|B|$-grid $\mathcal{G}$.

The construction of the grid implies that $E_{a}$ and $F_{b}$ satisfy $\left|E_{a}\right|=|B|$ and $\left|F_{b}\right|=|A|$ for all $a \in A$, and $b \in B$.

Diagonal-free grids appear whenever two arcs of two hypergraphs are multiplied with respect to the Cartesian product. In this sense they generalize the chordless squares appearing as Cartesian products of arcs of undirected simple graphs. This suggests to generalize the relation $\delta[19,14]$ in the following way:

Definition. Let $H$ be a connected hypergraph. For $E, F \in \mathcal{E}(H)$ we say $E$ and $F$ are in relation $\delta, E \delta F$, if one of the following conditions is satisfied: 


\section{CARTESIAN PRODUCT OF HYPERGRAPHS 9}

(i) $E$ and $F$ have no vertex in common and form the opposite arcs of a 4-cycle.

(ii) $E$ and $F$ are incident to at least one common vertex and there is no grid without diagonals that contains both $E$ and $F$.

(iii) $E=F$.

Note that whenever $E$ and $F$ share two or more vertices there is no $(|E| \times|F|)$-grid that contains $E$ and $F$, and hence $E \delta F$.

Obviously, the $\delta$ is reflexive and symmetric. Its transitive closure $\delta^{*}$, i.e., the smallest transitive relation containing $\delta$, is therefore an equivalence relation.

Condition (ii) implies that any two incident arcs $E$ and $F$ with $E \phi F$ span an $(|E| \times$ $|F|$ )-grid without diagonals, whose arcs we denote by $E,\left\{E_{a}\right\}_{a \in A}$ and $F,\left\{F_{b}\right\}_{b \in B}$, where $E \delta E_{a}$ and $F \delta F_{b}$ for all $a \in A$ and $b \in B$, respectively.

Lemma 4. Two incident arcs that are not in relation $\delta$ span a unique $(|E| \times|F|)$-grid.

Proof. Suppose there exists another $(|E| \times|F|)$-grid consisting of arcs $E,\left\{E_{a}^{\prime}\right\}_{a \in A}$ and $F,\left\{F_{b}^{\prime}\right\}_{b \in B}$. Then there must be a $k \in A$ and an $l \in B$ such that $E_{k}^{\prime} \notin\left\{E_{a}\right\}_{a \in A}$ and $F_{l}^{\prime} \notin\left\{F_{b}\right\}_{b \in B}$, respectively. Hence there exists both an arc $E_{r} \in\left\{E_{a}\right\}_{a \in A}$ and and arc $F_{s} \in\left\{F_{b}\right\}_{b \in B}$ such that $E_{k}^{\prime}$ and $E_{r}$ have common vertices and $F_{l}^{\prime}$ and $F_{s}$ have common vertices. Thus there is a 4 -cycle $E_{k}^{\prime} E_{r} F_{s} F_{l}^{\prime}$, where $E_{k}^{\prime}$ and $F_{s}$ as well as $E_{r}$ and $F_{l}^{\prime}$ are opposite arcs. Hence $E_{k}^{\prime} \delta F_{s}$ and $E_{r} \delta F_{l}^{\prime}$, and therefore $(E, F) \in \delta^{*}$. It follows that if $E$ and $F$ belong to distinct $\delta^{*}$-equivalence classes, they span a unique $(|E| \times|F|)$-grid.

This observation suggests the following definition:

Definition. Let $\gamma$ be an equivalence relation on the $\operatorname{arc}$ set $\mathcal{E}(H)$ of a hypergraph $H$. We say $\gamma$ has the grid property if any two adjacent arcs $E$ and $F$ of $H$ with $E \not / F$ span exactly one diagonal-free $|E| \times|F|$-grid.

Our discussion above implies that $\delta^{*}$ has the grid property.

Let $\gamma$ be an arbitrary equivalence relation on the arc set of a hypergraph $H$ that contains $\delta^{*}$. For any two $\operatorname{arcs} E$ and $F$ with $E \not / F$ we also have $E \phi^{*} F$ and therefore, they span exactly one (diagonal-free) $|E| \times|F|$-grid. As a consequence, every equivalence relation $\gamma$ that contains $\delta^{*}$ satisfies the grid property.

Lemma 5. Let $H$ be a connected hypergraph and let $\gamma$ be an equivalence relation on $\mathcal{E}(H)$ satisfying the grid property. Denote the equivalence classes of $\gamma$ by $\Gamma_{i}, i \in I$. Then every vertex of $v \in V(H)$ is incident to an $\operatorname{arc} E \in \Gamma_{i}$ for every $i \in I$.

Proof. Suppose that there is an equivalence class $\Gamma_{i}$ of $\gamma$ and a set of vertices that is not contained in any $\Gamma_{i}$-arc. By connectedness of $H$, there is a pair of vertices $u, v \in V(H)$ and an $\operatorname{arc} E \in \mathcal{E}(H)$ with $\{u, v\} \subseteq T(E) \cup H(E)$ such that $u$ belongs to a $\Gamma_{i}$-arc, say $F$, and there is no $\Gamma_{i}$-arc containing $v$. It follows that $E \notin \Gamma_{i}$, i.e., it must be contained in some other equivalence class $\Gamma_{k}, k \neq i$. By construction, $E$ and $F$ are two incident arcs 
belonging to different equivalence classes of $\gamma$ and hence span a grid. Thus there must be a $\Gamma_{i}$-arc containing $v$, contradicting the assumption.

Lemma 6. Let $H=\square_{i \in I}^{a} H_{i}$ be a weak Cartesian product of prime hypergraphs $H_{i}$ and let $E, F \in \mathcal{E}(H)$. If $E$ and $F$ are in relation $\delta$, they are in relation $\sigma$.

Proof. Suppose first, that for the $\operatorname{arcs} E$ and $F$ holds $E \cap F=\emptyset$ and there are arcs $E^{\prime}, F^{\prime} \in \mathcal{E}(H)$ such that $\left\{E, E^{\prime}, F, F^{\prime}\right\}$ is a 4-cycle. Moreover, we denote with $c(E)$ the coordinates where the vertices of the arc $E$ differ. W.l.o.g. assume $x_{1}$ is common to $E$ and $E^{\prime}, x_{2}$ is common to $E$ and $F^{\prime}, y_{1}$ is common to $F$ and $E^{\prime}$, and $y_{2}$ is common to $F$ and $F^{\prime}$. The coordinates varying within these arcs are denote by $c(E)=i, c(F)=j$, $c\left(E^{\prime}\right)=i^{\prime}, c\left(F^{\prime}\right)=j^{\prime}$; see Fig. 2 .

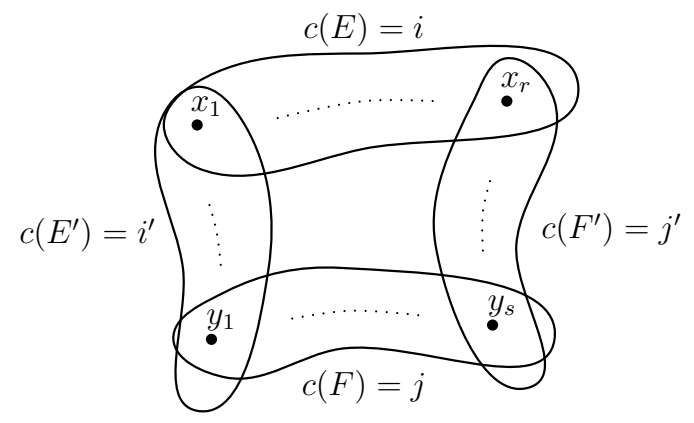

FIGURE 2. 4-cycle $E E^{\prime} F F^{\prime}$

Then we have:

$$
\begin{array}{ll}
p_{k}\left(x_{1}\right)=p_{k}\left(x_{2}\right) & \text { for all } k \neq i \\
p_{k}\left(y_{1}\right)=p_{k}\left(y_{2}\right) & \text { for all } k \neq j \\
p_{k}\left(x_{1}\right)=p_{k}\left(y_{1}\right) & \text { for all } k \neq i^{\prime} \\
p_{k}\left(x_{2}\right)=p_{k}\left(y_{2}\right) & \text { for all } k \neq j^{\prime} .
\end{array}
$$

It follows from (2) and (5) that

$$
p_{k}\left(x_{1}\right)=p_{k}\left(y_{2}\right) \quad \text { for all } k \neq i, j^{\prime}
$$

and from (3) and (4)

$$
p_{k}\left(x_{1}\right)=p_{k}\left(y_{2}\right) \quad \text { for all } k \neq j, i^{\prime} .
$$

Therefore we have either $i=j$ and $i^{\prime}=j^{\prime}$ or $i=i^{\prime}$ and $j^{\prime}=j$. Assume $i \neq j$. Then the latter case must hold and we have $p_{k}\left(x_{2}\right)=p_{k}\left(x_{1}\right)=p_{k}\left(y_{2}\right)$ for all $k \neq i$, and since 
$x_{2} \neq y_{1}$ holds, $p_{i}\left(x_{2}\right) \neq p_{i}\left(y_{1}\right)=p_{i}\left(y_{2}\right)$ and $p_{j}\left(x_{2}\right) \neq p_{j}\left(y_{2}\right)$. Hence, $x_{2}$ and $y_{2}$ differ in more than one coordinate, thus they cannot lie in the same arc $F^{\prime}$, which contradicts the assumption, therefore, $i=j$ must hold, i.e. $c(E)=c(F)$, and therefore $E \sigma F$.

Now let $E$ and $F$ be incident arcs of a hypergraph $H$ and assume that there is no $|E| \times|F|$-grid without diagonals containing them.

First, consider the case that $E$ and $F$ share more than a single vertex. Then there is an index $i \in I$ such that $\left|p_{i}(E)\right|>1$ and in particular, $\left|p_{i}\left(E^{\prime}\right)\right|>1$ holds for all $E^{\prime} \subseteq T(E) \cup H(E)$ with $\left|E^{\prime}\right|>1$.

Since $E$ and $F$ have more than one vertex in common it follows that $\mid p_{i}((T(E) \cup$ $H(E)) \cap(T(F) \cup H(F)) \mid>1$ and hence $\left|p_{i}(E)\right|>1$ and $\left|p_{i}(F)\right|>1$, and thus $E \sigma F$.

Now suppose that $E$ and $F$ share a single vertex $v$ and assume that $E$ and $F$ are not in relation $\sigma$. Let $\hat{E}=T(E) \cup H(E)=\{v\} \cup \bigcup_{a \in A}\left\{x_{a}\right\}$ and $\hat{F}=T(F) \cup H(F)=$ $\{v\} \cup \bigcup_{b \in B}\left\{y_{b}\right\}$. Furthermore set $j=c(F)$ and observe that $j \neq i$. For all $x_{a} \in E$, $a \in A$, and all $b \in B$ there exist vertices $z_{a b} \in V(H)$ such that

$$
\begin{aligned}
& p_{i}\left(z_{a b}\right)=p_{i}\left(x_{a}\right) \\
& p_{k}\left(z_{a b}\right)=p_{k}\left(y_{b}\right)
\end{aligned} \quad \text { for all } k \neq i .
$$

Using (9) and the fact, that $\left\{v, y_{b}\right\} \subseteq F$ for all $b \in B$ and $\left\{v, x_{a}\right\} \subseteq E$, we can conclude that the set $\hat{F}_{a}=\left\{x_{a}\right\} \cup \bigcup_{b \in B}\left\{z_{a b}\right\}$ satisfies

$$
p_{j}\left(\hat{F}_{a}\right)=\left\{p_{j}\left(x_{a}\right)\right\} \cup \bigcup_{b \in B}\left\{p_{j}\left(z_{a b}\right)\right\}=\left\{p_{j}(v)\right\} \cup \bigcup_{b \in B}\left\{p_{j}\left(y_{b}\right)\right\}=p_{j}(\hat{F})
$$

as well as

$$
p_{k}\left(z_{a b}\right)=p_{k}\left(y_{b}\right)=p_{k}(v)=p_{k}\left(x_{a}\right) \quad \text { for all } k \neq i, j \quad \text { and for all } b \in B
$$

Now we use (8) and (11) and obtain

$$
p_{k}\left(z_{a b}\right)=p_{k}\left(x_{a}\right) \quad \text { for all } k \neq i \quad \text { and for all } b \in B \text {. }
$$

Analgously, the set $\hat{E}_{b}=\left\{y_{b}\right\} \cup \bigcup_{a \in A}\left\{z_{a b}\right\}$ satisfies

$$
p_{i}\left(\hat{E}_{b}\right)=\left\{p_{i}\left(y_{b}\right)\right\} \cup \bigcup_{a \in A}\left\{p_{i}\left(z_{a b}\right)\right\}=\left\{p_{i}(v)\right\} \cup \bigcup_{a \in A}\left\{p_{i}\left(x_{a}\right)\right\}=p_{i}(\hat{E}) .
$$

Again, we use (8) and the fact that $\left\{v, y_{b}\right\} \subseteq F$ for all $b \in B$. 


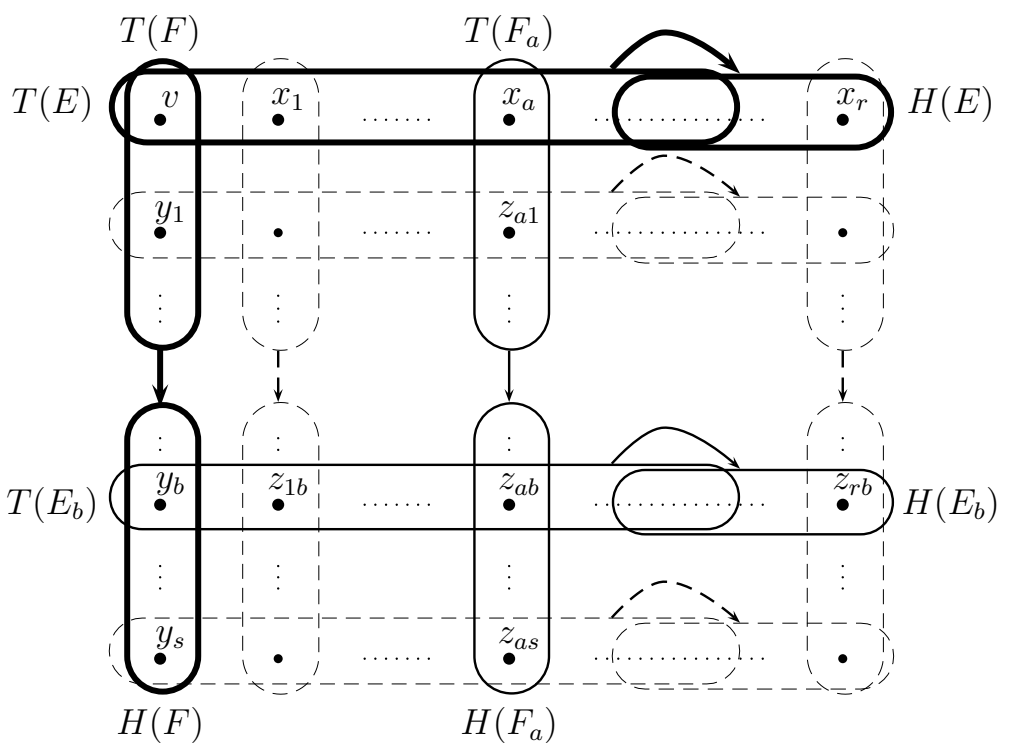

FIGURE 3. Arcs $E$ and $F$ (thick arcs) with $(E, F) \notin \sigma$ and the $|E| \times|F|$-grid they span

It will be useful to relabel $v$ as $z_{00}, \hat{E}$ as $\hat{E}_{0}, \hat{F}$ as $\hat{F}_{0}$, the $x_{l}$ as $z_{l 0}$ for all $l \in A$, and the $y_{l}$ as $z_{0 l}$ for all $l \in B$. Then we have

$$
\hat{E}_{b} \cap \hat{F}_{a}=\left\{z_{a b}\right\}
$$

for all $a \in A_{0}:=A \cup\{0\}$ and $b \in B_{0}:=B \cup\{0\}$.

Since $z_{0 b} \in T(F)$ (resp. $H(F)$ ) if and only if $p_{j}\left(z_{0 b}\right) \in p_{j}\left(T(F)\right.$ ) (resp. $p_{j}(H(F))$ ) for all $b \in B_{0}$, we can conclude by the definition of the (weak) Cartesian product and from equations (10) and (12) that for all $\hat{F}_{a}, a \in A$, there exists an arc $F_{a}=\left(T\left(F_{a}\right), H\left(F_{a}\right)\right)$ with $\left|F_{a}\right|=|F|$ such that $\hat{F}_{a}=T\left(F_{a}\right) \cup H\left(F_{a}\right)$. More precisely, we have

$$
\begin{aligned}
& T\left(F_{a}\right)=\left\{z_{a b} \mid b \in B_{0} \text { and } z_{0 b} \in T(F)\right\}, \text { resp. } \\
& H\left(F_{a}\right)=\left\{z_{a b} \mid b \in B_{0} \text { and } z_{0 b} \in H(F)\right\}
\end{aligned}
$$

for all $a \in A$.

An analogous argument can be made for $\hat{E}_{b}$ : Since $z_{a 0} \in T(E)$ (resp. $H(E)$ ) if and only if $p_{i}\left(z_{a 0}\right) \in p_{i}(T(E))$ (resp. $p_{i}(H(E))$ ) for all $a \in A_{0}$, it follows from equations (9) and (13) that for all $\hat{E}_{b}, b \in B$, there exists an arc $E_{b}=\left(T\left(E_{b}\right), H\left(E_{b}\right)\right)$ with $\left|E_{b}\right|=|E|$ such that $\hat{E}_{b}=T\left(E_{b}\right) \cup H\left(E_{b}\right)$. More precisely, we have

$$
\begin{aligned}
& T\left(E_{b}\right)=\left\{z_{a b} \mid a \in A_{0} \text { and } z_{a 0} \in T(E)\right\}, \text { resp. } \\
& H\left(E_{b}\right)=\left\{z_{a b} \mid a \in A_{0} \text { and } z_{a 0} \in H(E)\right\}
\end{aligned}
$$

for all $b \in B$. 
Equation (14) immediately implies that the intersection of more then two arcs is empty.

Consequently, whenever two adjacent $\operatorname{arcs} E$ and $F$ are not in relation $\sigma$, they span an $|E| \times|F|$-grid with $|E| \times|F|$ vertices.

It remains to show that these grids have no diagonals. Therefore, we need to show that there is no $D \in \mathcal{E}(H)$ such that $\left\{z_{a b}, z_{a^{\prime} b^{\prime}}\right\} \subseteq D$ for all $a, a^{\prime} \in\{0\} \cup A, b, b^{\prime} \in\{0\} \cup B$ with $a \neq a^{\prime}$ and $b \neq b^{\prime}$. For $z_{a b}, z_{a^{\prime} b^{\prime}}$ we have:

$$
\begin{aligned}
& p_{i}\left(z_{a b}\right) \stackrel{(8)}{=} p_{i}\left(z_{a 0}\right) \neq p_{i}\left(z_{a^{\prime} 0}\right) \stackrel{(8)}{=} p_{i}\left(z_{a^{\prime} b^{\prime}}\right) \\
& p_{j}\left(z_{a b}\right) \stackrel{(9)}{=} p_{j}\left(z_{0 b}\right) \neq p_{j}\left(z_{0 b^{\prime}}\right) \stackrel{(9)}{=} p_{j}\left(z_{a^{\prime} b^{\prime}}\right)
\end{aligned}
$$

The inequalities follow from the fact, that $\left\{z_{a 0}, z_{a^{\prime} 0}\right\} \subseteq E_{0}$ and $\left\{z_{0 b}, z_{0 b^{\prime}}\right\} \subseteq F_{0}$.

That means that $z_{a b}$ and $z_{a^{\prime} b^{\prime}}$ differ in more than one coordinate, hence, they cannot be contained in the same arc, which completes the proof.

Lemma 6 implies that $\delta \subseteq \sigma$ and since $\sigma$ is an equivalence relation, $\delta^{*} \subseteq \sigma$ holds as well. Thus, the product relation $\sigma$ has the grid property.

\section{CONVEXITY}

Lemma 7. Let $H=\square_{i \in I}^{a} H_{i}$ be a weak Cartesian product of connected hypergraphs $H_{i}$. Then each $H_{J}=\square_{j \in J}^{a} H_{j}$-layer is convex for any index set $J \subseteq I$.

Proof. It suffices to show that, whenever there is a path $P$ between two arbitrary vertices $u$ and $v$ of the same $H_{J}$-layer $H_{J}^{u}$ containing no $\operatorname{arcs}$ of this layer, then there exists a path $Q$ which entirely lies in $H_{J}^{u}$ and satisfies $|Q|<|P|$.

Suppose $P=\left(u=u_{0}, E_{1}, u_{1}, E_{2}, \ldots, u_{k-1}, E_{k}, u_{k}=v\right)$. Since $u$ and $v$ belong to the same $H_{j}$-layer, $p_{l}(u)=p_{l}(v)$ holds for all $l \in I \backslash J$. There must be an arc $E_{i}$ of $P$ such that $E_{i}$ is contained in some $H_{J}$ layer, by assumption different from $H_{J}^{u}$. Otherwise we would have $p_{l}(u)=p_{l}(v)$ for all $l \in J$, hence $p_{l}(u)=p_{l}(v)$ for all $l \in I$, i.e. $u=v$.

Let $\left\{E_{j_{1}}, \ldots, E_{j_{r}}\right\}$ be a subset of $\operatorname{arcs}$ of $P$, with $j_{1}, j_{2}, \ldots, j_{r} \in\{1, \ldots, k\}, j_{1}<j_{2}<$ $\ldots<j_{r}$, that are in some $H_{J}$-layer different from $H_{J}^{u}$, and no arc is the copy of another. To be more precise, for each $j_{i}$ there is a $k_{i} \in J$ with

$$
p_{k_{i}}\left(E_{j_{i}}\right) \in \mathcal{E}\left(H_{k_{i}}\right) \quad \text { and } \quad p_{k_{a}}\left(E_{j_{a}}\right) \neq p_{k_{b}}\left(E_{j_{b}}\right) \quad \text { for } a \neq b
$$

and $j_{r}$ is maximal. Note that $a \neq b$ does not imply $k_{a} \neq k_{b}$. 


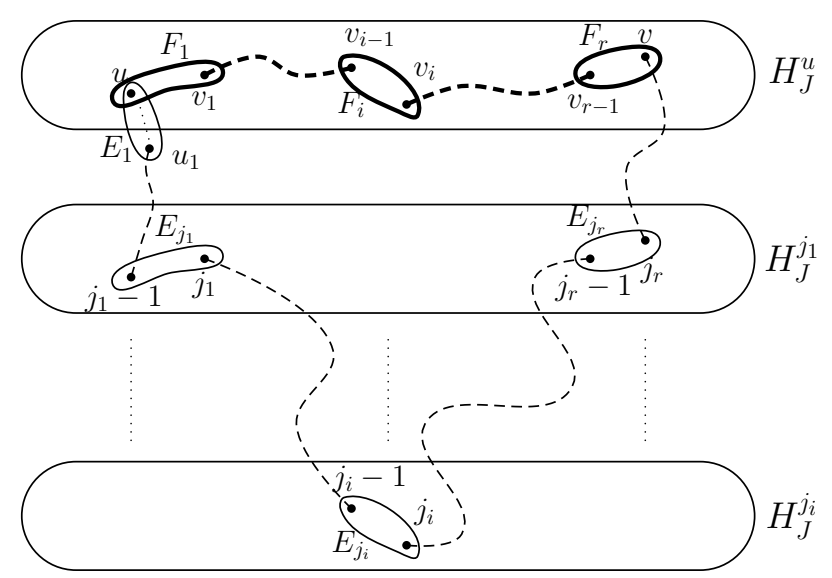

FIGURE 4. Idea of the proof: Path $P$ and Path $Q$ (thick) which we got by shifting the $\operatorname{arcs} E_{j_{i}}$ in the $H_{J}^{u}$-layer

By assumption, $E_{1}$ is not contained in any $H_{J}$-layer, thus $r<k$. Without loss of generality, we can assume that the $E_{j_{i}}$ are not incident. In the following we will denote the vertices $u_{j_{i}}$ by $j_{i}$. If we set $j_{0}:=u_{0}$, we have

$$
p_{l}\left(j_{i-1}\right)=p_{l}\left(j_{i}-1\right) \quad \text { for all } l \in J \text { and all } i \in\{1, \ldots, r\}
$$

and since $j_{i}-1, j_{i} \in E_{j_{i}}$,

$$
p_{l}\left(j_{i}\right)=p_{l}\left(j_{i}-1\right)
$$

holds for all $l \neq k_{i}, k_{i} \in J$. Furthermore, for each $j_{i}, i \in\{1, \ldots, r\}$, there exists a $v_{i} \in V(H)$, such that

$$
\begin{aligned}
& p_{l}\left(v_{i}\right)=p_{l}(u) \quad \text { for all } l \in I \backslash J \\
& p_{l}\left(v_{i}\right)=p_{l}\left(j_{i}\right) \quad \text { for all } l \in J
\end{aligned}
$$

In particular, Equation (22) implies $v_{i} \in V\left(H_{J}^{u}\right)$ for all $i \in\{1, \ldots, r\}$. It follows

$$
p_{l}\left(v_{i-1}\right) \stackrel{(23)}{=} p_{l}\left(j_{i-1}\right) \stackrel{(20),(21)}{=} p_{l}\left(j_{i}\right) \stackrel{(23)}{=} p_{l}\left(v_{i}\right) \quad \text { for all } l \in J \backslash\left\{k_{i}\right\}
$$

and by Equation (22) we have

$$
p_{l}\left(v_{i-1}\right)=p_{l}\left(v_{i}\right) \quad \text { for all } l \in I \backslash J
$$

hence by Equations (24) and (25)

$$
p_{l}\left(v_{i-1}\right)=p_{l}\left(v_{i}\right) \quad \text { for all } l \neq k_{i}
$$


In other words, any two vertices $v_{i-1}, v_{i}$ lie in the same $H_{k_{i}}$ layer for some $k_{i} \in J$.

Next we show that there are $\operatorname{arcs} F_{i} \in \mathcal{E}\left(H_{J}^{u}\right)$ containing both $v_{i-1}$ and $v_{i}$. From Equations (20) and (23) it follows

$$
\begin{aligned}
p_{k_{i}}\left(v_{i-1}\right) & \stackrel{(23)}{=} p_{k_{i}}\left(j_{i-1}\right) \stackrel{(20)}{=} p_{k_{i}}\left(j_{i}-1\right) \\
p_{k_{i}}\left(v_{i}\right) & \stackrel{(23)}{=} p_{k_{i}}\left(j_{i}\right)
\end{aligned}
$$

Thus we have by Equations (27), (28) and (19)

$$
p_{k_{i}}\left(v_{i-1}\right), p_{k_{i}}\left(v_{i}\right) \in p_{k_{i}}\left(E_{j_{i}}\right)
$$

Hence by Equations (22), (26) and (29), for each $i \in\{1, \ldots, r\}$ there exists an arc $F_{i}$ in $H_{J}^{u}$, such that $v_{i-1}, v_{i} \in F_{i}$.

Now consider the vertex $v_{r}$. Since there is no more arc $E_{j}, j>j_{r}$, of $P$ that is contained in any $H_{J}$-layer, $j_{r}$ and $v$ belong to the same $\widehat{H_{J}}:=\square_{i \in I \backslash J} H_{i}$-layer Therefore we have

$$
p_{l}\left(v_{r}\right) \stackrel{(23)}{=} p_{l}\left(j_{r}\right)=p_{l}(v) \quad \text { for all } l \in J
$$

and from the definition of $v_{r}$ and the fact that $u$ and $v$ are in the same $H_{J}$-layer, it follows

$$
p_{l}\left(v_{r}\right) \stackrel{(22)}{=} p_{l}(v) \quad \text { for all } l \in I \backslash J .
$$

Therefore, we can conclude $v_{r}=v$, and we have found a path $Q=(u=$ $\left.v_{0}, F_{1}, v_{1}, \ldots, v_{r-1}, F_{r}, v_{r}=v\right)$ from $u$ to $v$, whose arcs all lie entirely within $H_{J}^{u}$. Furthermore, we have $|Q|=r<k=|P|$, which completes the proof.

Definition. An equivalence relation $\gamma$ on the arc set $\mathcal{E}(H)$ of a connected hypergraph $H$ with equivalence classes $\Gamma_{i}, i \in I$, is called convex if for any $J \subseteq I$ every connected component of the partial hypergraph generated by $\bigcup_{i \in J} \Gamma_{i}$ is convex.

By Lemma 7, the product relation $\sigma$ is a convex relation. Moreover, any product relation must be convex and has to satisfy the grid property.

Lemma 8. Let $\gamma$ be an equivalence relation on the $\operatorname{arc}$ set $\mathcal{E}(H)$ of a connected hypergraph $H$ which satisfies the grid property. Let $\Gamma$ be an equivalence class of $\gamma$. If all connected components of the partial hypergraph of $H$ generated by $\Gamma$ are convex, they are isomorphic.

Proof. Let $H_{\Gamma}$ be the partial hypergraph generated by $\Gamma$ with connected components $C_{i}, i \in I$ and let $\widehat{\Gamma}$ denote the union of all equivalence classes of $\gamma$, distinct from $\Gamma$, i.e., 
$\widehat{\Gamma}=\bigcup_{\Gamma^{\prime} \neq \Gamma} \Gamma^{\prime}$. It suffices to show that any two components $C_{1}$ and $C_{2}$ that are connected by a $\widehat{\Gamma}$-arc are isomorphic. We define a mapping $\varphi: V\left(C_{1}\right) \rightarrow V\left(C_{2}\right)$ such that $x \mapsto \varphi x$, whenever $x$ and $\varphi x$ are connected by a $\widehat{\Gamma}$-arc. From the grid property and Lemma 5 , it follows that for all $x \in V\left(C_{1}\right)$ there exists a $\varphi x \in V\left(C_{2}\right)$. The grid property ensures that adjacent vertices in $C_{1}$ have different images in $C_{2}$ and arcs in $C_{1}$ map onto $\operatorname{arcs}$ in $C_{2}$, such that $\varphi(E)=\varphi((T(E), H(E)))=(T(\varphi(E)), H(\varphi(E))) \in \mathcal{E}\left(C_{2}\right)$ for all $E \in \mathcal{E}\left(C_{1}\right)$. Convexity implies that non adjacent vertices in $C_{1}$ have different images in $C_{2}$ as well, i.e., the mapping $\varphi$ is injective. On the other hand we can extend $\varphi^{-1}$ to a mapping $\psi: V\left(C_{2}\right) \rightarrow V\left(C_{1}\right)$. Analogously, it follows that for all $y \in V\left(C_{2}\right)$ there is a $\psi y$ in $V\left(C_{1}\right)$, hence $\varphi^{-1}=\psi$, i.e., $\varphi$ is bijective, and every arc in $C_{2}$ maps onto an $\operatorname{arc}$ in $C_{1}$, thus $\varphi$ is an isomorphism between $C_{1}$ and $C_{2}$.

Sometimes, the transitive closure of $\delta$ is already convex. If this is the case, then each path between two vertices of the same connected component of an equivalence class of $\delta^{*}$ must contain at least one arc of this equivalence class as a consequence of Lemma 7 .

Lemma 9. Let $\gamma$ be an equivalence relation on the $\operatorname{arc} \operatorname{set} \mathcal{E}(H)$ of a connected hypergraph $H$ satisfying the grid property with only two equivalence classes $\Gamma$ and $\widehat{\Gamma}$. Let $H_{\Gamma}$ and $H_{\widehat{\Gamma}}$ be the partial hypergraphs generated by $\Gamma$ and $\widehat{\Gamma}$, with connected components $C_{i}, i \in I$, and $\widehat{C}_{j}, j \in J$, respectively. Then

$$
V\left(C_{i}\right) \cap V\left(\widehat{C}_{j}\right) \neq \emptyset \quad \text { for all } i \in I, j \in J .
$$

In particular,

$$
\left|V\left(C_{i}\right) \cap V\left(\widehat{C}_{j}\right)\right|=1
$$

holds if $C_{i}$ and $\widehat{C}_{j}$ are convex.

Proof. Suppose there are components $C_{i}, \widehat{C}_{j}$ with $V\left(C_{i}\right) \cap V\left(\widehat{C}_{j}\right)=\emptyset$, such that they have minimal distance. Let $P=\left(v_{0}, E_{1}, v_{1}, E_{2}, \ldots, E_{k}, v_{k}\right)$ be a shortest path from $C_{i}$ to $\widehat{C}_{j}$, such that $v_{0} \in V\left(C_{i}\right)$ and $v_{k} \in V\left(\widehat{C}_{j}\right)$. Obviously, the first arc $E_{1}$ must lie in $\widehat{\Gamma}$ and the vertex $v_{1}$ is not in $C_{i}$, otherwise $E_{1}$ would be in $\Gamma$ which contradicts the minimality of $P$. Lemma 5 implies that $v_{1}$ must be contained in a $\Gamma$-component, say $C_{k}$. Since the distance from $C_{k}$ to $\widehat{C}_{j}$ is smaller than $|P|$, we have $V\left(C_{k}\right) \cap V\left(\widehat{C}_{j}\right) \neq \emptyset$. Let $w$ be a vertex in $V\left(C_{k}\right) \cap V\left(\widehat{C}_{j}\right)$ and let $P^{\prime}$ be a path from $v_{1}$ to $w$ in $C_{k}$. By repeated application of the grid property we obtain a vertex $u$ in $V\left(C_{i}\right)$ connected to $w$ by a $\widehat{\Gamma}$-arc. But then $u$ must be in $V\left(\widehat{C}_{j}\right)$ and thus $\left|V\left(C_{i}\right) \cap V\left(\widehat{C}_{j}\right)\right| \geq 1$.

Now assume $\left|V\left(C_{i}\right) \cap V\left(\widehat{C}_{j}\right)\right| \geq 2$. Let $u, w \in V\left(C_{i}\right) \cap V\left(\widehat{C}_{j}\right)$. By connectivity we have a path $Q$ from $u$ to $w$ in $C_{i}$ and a path $Q^{\prime}$ from $u$ to $w$ in $\widehat{C}_{j}$ as well. Therefore 
CARTESIAN PRODUCT OF HYPERGRAPHS 17

either $|Q|>\left|Q^{\prime}\right|$ or $\left|Q^{\prime}\right|>|Q|$ or $|Q|=\left|Q^{\prime}\right|$ holds. Hence either $C_{i}$ or $\widehat{C}_{j}$ or both are not convex, and thus the second assertion holds.

\section{PROOF OF THE THEOREMS}

We are now able to prove Theorem 1:

Proof of Theorem 1. First assume that $\gamma$ has only two equivalence classes $\Gamma$ and $\widehat{\Gamma}$ with connected components $C_{i}, i \in J$ and $\widehat{C}_{j}, j \in \widehat{J}$ respectively, of the generated partial hypergraphs. By Lemma 9 we can assign uniquely determined coordinates $(i, j)$ to each vertex of $H$, whenever $\{v\}=V\left(C_{i}\right) \cap V\left(\widehat{C}_{j}\right), i \in J, j \in \widehat{J}$. On the other hand for all such coordinates there exists a uniquely determined vertex in $V(H)$, since $\left|V\left(C_{i}\right) \cap V\left(\widehat{C}_{j}\right)\right|=1$.

In the following we will identify each vertex of $H$ with its coordinates. Obviously we have $V\left(C_{i}\right)=\{(i, j) \mid j \in \widehat{J}\}$ for all $i \in J$ and $V\left(\widehat{C}_{j}\right)=\{(i, j) \mid i \in J\}$ for all $j \in \widehat{J}$. Recall that Lemma 8 implies that the $C_{i}$ are isomorphic for all $i \in J$. In particular $C_{1} \simeq C_{i}$ holds for all $i \in J$. The isomorphism is given by the mapping

$$
(1, j) \mapsto(i, j) \text { for all } j \in \widehat{J} .
$$

If $C_{1}$ and $C_{i}$ are connected by an arc, it is an isomorphism as in the proof of Lemma 8. If they are connected by a path, it is an isomorphism by induction on the length of the path. Analogously we have $\widehat{C}_{1} \simeq \widehat{C}_{j}$ for all $j \in \widehat{J}$ given by the isomorphism

$$
(i, 1) \mapsto(i, j) \text { for all } i \in J .
$$

A set of vertices $E=\left\{\left(i_{1}, j_{1}\right), \ldots,\left(i_{q}, j_{q}\right), \ldots\right\}, i_{1}, \ldots, i_{q}, \ldots \in J, j_{1}, \ldots, j_{q}, \ldots \in$ $\widehat{J}, \quad$ is an arc in $H$ with $T(E)=\left\{\left(i_{t_{1}}, j_{t_{1}}\right), \ldots,\left(i_{t_{r}}, j_{t_{r}}\right), \ldots\right\}$ and $H(E)=$ $\left\{\left(i_{h_{1}}, j_{h_{1}}\right), \ldots,\left(i_{h_{s}}, j_{h_{s}}\right), \ldots\right\}$ if and only if either

(i) it is in the same $C_{i}$, hence $i_{1}=\ldots=i_{q}=\ldots=i$ and $E_{1}=\left\{\left(1, j_{1}\right), \ldots,\left(1, j_{q}\right), \ldots\right\}$ is an arc in $C_{1}$, such that $T\left(E_{1}\right)=\left\{\left(1, j_{t_{1}}\right), \ldots,\left(1, j_{t_{r}}\right), \ldots\right\}$ and $H\left(E_{1}\right)=$ $\left\{\left(1, j_{h_{1}}\right), \ldots,\left(1, j_{h_{s}}\right), \ldots\right\}$, or

(ii) it is in the same $\widehat{C}_{j}$, hence $j_{1}=\ldots=j_{q}=\ldots=j$ and $E_{2}=\left\{\left(i_{1}, 1\right), \ldots,\left(i_{q}, 1\right)\right\}$ is an arc in $\widehat{C}_{1}$, such that $T\left(E_{2}\right)=\left\{\left(i_{t_{1}}, 1\right), \ldots,\left(i_{t_{r}}, 1\right), \ldots\right\}$ and $H\left(E_{2}\right)=$ $\left\{\left(i_{h_{1}}, 1\right), \ldots,\left(i_{h_{s}}, 1\right), \ldots\right\}$.

That is, $H$ is isomorphic to $C_{1} \square \widehat{C}_{1}$.

Now define hypergraphs $H_{1}$ and $H_{2}$ by setting $V\left(H_{1}\right)=\left\{i:(i, 1) \in V\left(C_{1}\right)\right\}$ and $V\left(H_{2}\right)=\left\{j:(1, j) \in V\left(\widehat{C}_{1}\right)\right\} . H_{1}$ and $H_{2}$ are isomorphic to $C_{1}$ and $\widehat{C}_{1}$ by the isomorphic mappings $i \mapsto(i, 1)$ and $j \mapsto(1, j)$ respectively, thus $H=H_{1} \square H_{2}$.

Assume now that $\gamma$ has arbitrarily many equivalence classes $\Gamma_{i}, i \in I$. Let $\gamma_{i}$ be the equivalence relation with the two equivalence classes $\Gamma_{i}$ and $\widehat{\Gamma}_{i}=\bigcup_{k \in I, k \neq i} \Gamma_{k}$ for 
arbitrary $i \in I$. As already shown, we get a factorization of $H$ into two factors $H_{i} \square \widehat{H}_{i}$ where $H_{i}$ and $\widehat{H}_{i}$ belong to $\Gamma_{i}$ and $\widehat{\Gamma}_{i}$, respectively. We will call the projection, more precisely the image of the projection, of a vertex $v$ in $H_{i} \square \widehat{H}_{i}$ into the factor $H_{i}$ the $i$-th coordinate of $v$, denoted by $v^{i}$.

Clearly, we can assign coordinates to each vertex. If two vertices $u, v$ have the same $i$-th coordinate, then, by convexity, there is no $\Gamma_{i}$-arc on any shortest path between them. Thus, if $u$ and $v$ have the same coordinates, there is no nontrivial shortest path between them, hence $u=v$. It follows that the assignment of coordinates to vertices of a connected hypergraph $H$ is bijective.

A subset $E=\left\{v_{1}, \ldots, v_{q}, \ldots\right\}$ of $V(H)$ is an arc of $H$ with $T(E)=\left\{v_{t_{1}}, \ldots, v_{t_{r}}, \ldots\right\}$ and $H(E)=\left\{v_{h_{1}}, \ldots, v_{h_{s}}, \ldots\right\}$ if and only if the $v_{l}$ differ in the same coordinate, say the $i$-th, for all $l \in\{1, \ldots, q, \ldots\}$ and $E_{i}=\left\{v_{1}^{i}, \ldots, v_{q}^{i}, \ldots\right\}$ is an arc in $H_{i}$ with $T\left(E_{i}\right)=$ $\left\{v_{t_{1}}^{i}, \ldots, v_{t_{r}}^{i}, \ldots\right\}$ and $H\left(E_{i}\right)=\left\{v_{h_{1}}^{i}, \ldots, v_{h_{s}}^{i}, \ldots\right\}$.

Since $H$ is connected, its vertices differ in at most finitely many coordinates, thus we have $H \simeq \square_{i \in I}^{a} H_{i}$ for any $a \in V(H)$.

The equivalence relation whose only equivalence class is the entire arc set of a hypergraph $H$ is trivially convex and satisfies the grid property and is therefore a product relation. This relation always exists. By Theorem 1 we can conclude that any convex relation on the arc set of a connected hypergraph that satisfies the grid property is a product relation and induces a factorization of this hypergraph. The smallest convex relation satisfying the grid-property, if such a relation exists at all, therefore must induce a PFD with respect to the Cartesian product.

The next lemma is well known for undirected graphs. Its proof is one of the key application of the square-property [14]. This proof step can directly be generalized to the grid-property in the case of hypergraphs. Moreover, since the definition of a path (i.e., a weak path) coincides with the definition of a path in the undirected case, we can immediately state the next lemma for hypergraphs.

Lemma 10. Let $\gamma_{j}, j \in J$ be an arbitrary set of convex relations on the arc set $\mathcal{E}(H)$ of a hypergraph $H$ containing $\delta$. Then $\gamma=\bigcap_{j \in J} \gamma_{j}$ is convex.

It is clear that for arbitrary equivalence relations on the arc set of a hypergraph, which satisfy the grid-property, their intersection also has the grid-property. Therefore Lemma 10 implies that there is exactly one finest convex equivalence relation on the arc set $\mathcal{E}(H)$ of a hypergraph $H$ satisfying the grid property, namely the intersection of all convex relations on $\mathcal{E}(H)$ containing $\delta$, that is its convex hull, $\mathcal{C}(\delta)$.

Conversely, any product relation must be convex and contains $\delta$. Thus we have proved the Theorems 2 and 3 . We conclude, furthermore

Corollary. The PFD of a connected hypergraph with respect to the Cartesian product is unique in the class of finite simple hypergraphs. 
CARTESIAN PRODUCT OF HYPERGRAPHS 19

\section{ACKNOWLEDGMENTS}

We thank Wilfried Imrich for many stimulating discussions on graph and hypergraph products.

\section{References}

[1] C. Berge. Hypergraphs: Combinatorics of finite sets. North-Holland, Amsterdam, NL, 1989.

[2] C. Berge and M. Simonovitis. The coloring numbers of the direct product of two hypergraphs. In Hypergraph Seminar, volume 411 of Lecture Notes in Mathematics, pages 21-33, Berlin, Heidelberg, 1974. Springer-Verlag.

[3] A. Bretto. Hypergraphs and the helly property. Ars Comb., 78:23-32, 2006.

[4] A. Bretto, Y. Silvestre, and T. Vallée. Cartesian product of hypergraphs: properties and algorithms. In 4th Athens Colloquium on Algorithms and Complexity (ACAC 2009), volume 4 of EPTCS, pages 22-28, 2009.

[5] W Dörfler. Multiple Covers of Hypergraphs. Annals N.Y. Acad. Sci., 319:169-176, 1979.

[6] W. Dörfler and D. A. Waller. A category-theoretical approach to hypergraphs. Arch. Math., 34:185-192, 1980.

[7] G. Gallo, G. Longo, S. Pallottino, and S. Nguyen. Directed hypergraphs and applications. Discrete Appl. Math., 42:177-201, 1993.

[8] G. Gallo and M. G. Scutellà. Directed hypergraphs as a modelling paradigm. Rivista AMASES, 21:97-123, 1998.

[9] W. Imrich. Kartesisches Produkt von Mengensystemen und Graphen. Studia Sci. Math. Hungar., 2:285-290, 1967.

[10] W. Imrich. über das schwache Kartesische Produkt von Graphen. J. Comb. Theory, 11:116, 1971.

[11] W. Imrich and S. Klavžar. Product Graphs: Structure and Recognition. John Wiley \& Sons, New York, 2000.

[12] W. Imrich, S. Klavžar, and D. F. Rall. Graphs and Their Cartesian Product. Topics in Graph Theory. A K Peters, Ltd., Wellesley, MA, 2008.

[13] W. Imrich and P. F. Stadler. A prime factor theorem for a generalized direct product. Discussiones Math. Graph Th., 26:135-140, 2006.

[14] W. Imrich and J. Žerovnik. Factoring Cartesian-product graphs. J. Graph Theory, 18:557567, 1994.

[15] S Klamt, U-U Haus, and F Theis. Hypergraphs and cellular networks. PLoS Comput Biol, 5:e1000385, 2009.

[16] D. J. Miller. Weak cartesian product of graphs. Colloq. Math., 21:55-74, 1970.

[17] D. Mubayi and V. Rödl. On the chromatic number and independence number of hypergraph products. J. Comb. Theory, Ser. B, 97:151-155, 2007. 
[18] R. Pemantle, J. Propp, and D. Ullman. On tensor powers of integer programs. SIAM J. Discrete Math., 5:127-143, 1992.

[19] G. Sabidussi. Graph multiplication. Math. Z., 72:446-457, 1960.

[20] X. Zhu. On the chromatic number of the product of hypergraphs. Ars Comb., 34:25-31, 1992.

\section{APPENDIX: BASIC DEFINITIONS}

A partial hypergraph $H^{\prime} \subseteq H$ of a hypergraph $H=(V, \mathcal{E})$ is a hypergraph $H^{\prime}=\left(V^{\prime}, \mathcal{E}^{\prime}\right)$ with $V^{\prime} \subseteq V$ and $\mathcal{E}^{\prime} \subseteq \mathcal{E}$. A partial hypergraph $H^{\prime}=\left(V^{\prime}, \mathcal{E}^{\prime}\right) \subseteq H=(V, \mathcal{E})$ is generated by the arc set $\mathcal{E}^{\prime}$ if $\bar{V}^{\prime}=\cup_{E \in \mathcal{E}^{\prime}} E$. It is induced by the vertex set $V^{\prime}, H^{\prime}=\left\langle V^{\prime}\right\rangle$, if $\mathcal{E}^{\prime}=\left(E \in \mathcal{E} \mid E \subseteq V^{\prime}\right)$

A strong path or simple path $P$ of length $k$ in $H=(V, \mathcal{E})$ is a sequence $P=$ $\left[v_{0}, E_{1}, v_{1}, E_{2}, \ldots, E_{k}, v_{k}\right]$ of distinct vertices and arcs of $H$, such that $v_{0} \in T\left(E_{1}\right)$, $v_{k} \in H\left(E_{k}\right)$ and $v_{j} \in H\left(E_{j}\right) \cap T\left(E_{j+1}\right)$. A weak path $P^{w}$ of length $k$ in a hypergraph $H=(V, \mathcal{E})$ is a sequence $P^{w}=\left(v_{0}, E_{1}, v_{1}, E_{2}, \ldots, E_{k}, v_{k}\right)$ of distinct vertices and arcs of $H$, such that $v_{0} \in T\left(E_{1}\right) \cup H\left(E_{1}\right), v_{k} \in T\left(E_{k}\right) \cup H\left(E_{k}\right)$ and $v_{j} \in\left[T\left(E_{j}\right) \cup H\left(E_{j}\right)\right] \cap\left[T\left(E_{j+1}\right) \cup H\left(E_{j+1}\right)\right]$. For the sake of convenience, we will refer to weak paths simply as paths. A hypergraph $H$ is said to be weakly connected or simply connected for short, if each two vertices of $H$ can be connected by a path. A path between two partial hypergraphs $H^{\prime}$ and $H^{\prime \prime}$ of a hypergraph $H$ is a path in $H$ between two vertices $v \in V\left(H^{\prime}\right)$ and $w \in V\left(H^{\prime \prime}\right)$.

The distance between two vertices of a hypergraph is the length of the shortest path connecting them. The distance $d_{H}\left(H^{\prime}, H^{\prime \prime}\right)$ between two partial hypergraphs $H^{\prime}, H^{\prime \prime}$ is the minimal length of a path between $H^{\prime}$ and $H^{\prime \prime}$. A partial hypergraph $H^{\prime} \subseteq H$ is convex if all shortest paths of $H$ between vertices of $H^{\prime}$ are contained in $H^{\prime}$.

A 4-cycle in $H$ is a partial hypergraph generated by $\operatorname{arcs} E_{1}, E_{2}, E_{3}$, and $E_{4}$, such that $E_{i} \cap E_{i+1} \neq \emptyset$, where the subscripts are taken modulo 4 .

A homomorphism from $H_{1}=\left(V_{1}, \mathcal{E}_{1}\right)$ into $H_{2}=\left(V_{2}, \mathcal{E}_{2}\right)$ is a mapping $\varphi: V_{1} \rightarrow V_{2}$ such that $\varphi(E)$ is an arc in $H_{2}$ whenever $E$ is an arc in $H_{1}$ with the property that $\varphi(T(E))=$ $T(\varphi(E))$ and $\varphi(H(E))=H(\varphi(E))$. A mapping $\varphi: V_{1} \rightarrow V_{2}$ is a weak homomorphism if arcs are mapped either on arcs or on vertices. A bijective homomorphism $\varphi$ whose inverse function is also a homomorphism is called an isomorphism. 